A Palabras clave/ Participación ciudadana, recorridos comentados, diagnóstico urbano, campus universitario.

A Keywords/ Citizen participation, commented visits, urban diagnostic, university campus.

$\Delta$ Recepción/ 5 enero 2018

$\triangle$ Aceptación/ 26 abril 2018

\title{
Participación ciudadana en
} movimiento: Metodología de recorridos comentados por la Universidad de Magallanes, Punta Arenas.

\section{Citizen participation in movement:}

Methodology of commented tours through

the Universidad de Magallanes, Punta Arenas.

\section{Karen Andersen}

Arquitecta, Universidad de Chile, Chile. Master d'Urbanisme et territoires, I.U.P., Université Paris XII, Francia.

Doctora en Aménagement de l'espace, Urbanisme, Université Paris Est, Francia. Académica, Instituto de Arquitectura y Urbanismo, Universidad Austral de Chile, Chile. kandersenc@gmail.com

\section{Sofía Balbontín}

Arquitecta, Pontificia Universidad Católica de Chile, Chile.

Académica, Facultad de Arquitectura, Diseño

y Construcción, Universidad de las Américas, Chile

RESUMEN/ Dentro de los estudios urbanos, las herramientas etnográficas propias de la investigación antropológica han resurgido, reinventándose en función de los intereses de la investigación sobre la cotidianeidad, la experiencia y las prácticas urbanas. Este artículo estudia las implicancias y resultados de una metodología de investigación en movimiento realizada en el marco del diseño del Plan Maestro de la Universidad de Magallanes, Punta Arenas, Chile. Se trata de una metodología de recorridos comentados, aplicada como parte de un proceso de participación ciudadana, que incorpora una mirada natural del espacio. El espacio se recorre y desde la percepción colectiva in situ, este se significa y valora. El objetivo de esta investigación es enriquecer las elaboraciones teóricas y prácticas de la planificación urbana en torno a la participación ciudadana en el marco del desarrollo de un diagnóstico urbano, con nuevas metodologías cualitativas que utilizan la experiencia como técnica de acceso a la información del lugar. ABSTRACT/ Ethnographic tools proper to anthropological research have reemerged within urban studies, reinventing themselves in response to the interests of investigations about urban daily life, experiences and practices. This article discusses the implications and findings of a research in movement methodology conducted under the design of the Master Plan of Universidad de Magallanes, Punta Arenas, Chile. This methodology involves commented visits applied as part of a citizen participation process that introduces a natural view to space. The space is visited and provided with meaning and value from a collective on-site perception. The purpose of this research is to enrich the theoretical and practical ideas of urban planning around citizen participation under the framework of an urban diagnostic, with new qualitative methodologies that use experience as a technique to access information about the place.

INTRODUCCIÓN. Los estudios urbanos han sido escenario de experimentación metodológica. Entre ellos encontramos desde los estudios sociológicos de Park en la Escuela de Chicago (Park 1999), los estudios sobre los comportamientos y prácticas en los espacios públicos urbanos (Augoyard 1979; Whyte 2001), hasta las derivas situacionistas de artistas, sociólogos y urbanistas (Debord 1975).
Siguiendo la teoría de la percepción, afirmaremos que la percepción es una función directa del estímulo y no una construcción a partir de una interpretación de datos sensoriales (Gibson 1979). A partir de esta idea, la percepción del espacio se organiza en la experiencia, y el caminar o el deambular como modo de vivenciar y de comprender la vida urbana se pone en el centro de algunas de estas nuevas 
metodologías de investigación que tienen como objetivo recoger la experiencia sensorial y afectiva de los espacios. Esta teoría será la base para la elaboración de una serie de metodologías para estudiar los espacios urbanos públicos y privados. Nuestra experiencia participativa en un campus universitario se apoyará en las elaboraciones teóricas y prácticas que se basan en la percepción y experiencia, incorporando, además, la participación de los usuarios (Grosjean y Thibaud 2001).

El recorrido comentado es la herramienta metodológica que guiará este trabajo de campo, el cual consiste en una recolección de información a partir de un paseo guiado por el lugar de estudio, a modo de entrevista individual o colectiva, para extraer información acerca de la vivencia de la comunidad en el espacio. El método se funda sobre la premisa de que el individuo entiende el mundo desde los sentidos, y el medio a través del cual aprecia la realidad, es la percepción. Jean-Paul Thibaud, sociólogo francés que estudia la teoría del ambiente urbano, se refiere al método del recorrido comentado o parcours commenté, como aquel que obtiene la información desde la percepción en movimiento y consiste en tres actos: caminar, percibir y describir (Thibaud 2001).

La metodología de recorridos comentados incorpora una mirada natural del espacio. Es el espacio utilizado, vivido y percibido el que nos interesa comprender a través de esta herramienta. La herramienta consiste en vivir la experiencia del espacio junto a grupos de usuarios, con el objetivo de 'contar el espacio' en la medida que se recorre. La experiencia siempre es única e irrepetible, anclada al momento, al lugar y a las personas que participan en el recorrido. Esto hace que los resultados de esta experiencia no sean tan relevantes en relación a la representatividad social de los relatos de los participantes, como por la profundidad y detalle de la información recopilada sobre la relación entre usuario y lugares habitados. La aplicación de la herramienta es de interés en la medida que se presenta como una técnica que observa la relación de los usuarios con el lugar in situ, y donde sus olores, sensaciones, colores y ruidos también participan, incentivando la palabra de los habitantes. Se estudiarán las implicancias y resultados de un recorrido comentado realizado en el marco del proceso participativo para el diseño del Plan Maestro de la Universidad de Magallanes (UMAG), Punta Arenas, Chile. El objetivo de esta investigación es abrir la reflexión en relación a herramientas de participación ciudadana que incorporen la percepción de los habitantes en la realización de diagnóstico urbanos. Esta experiencia profesional parte de la premisa que, para realizar un buen diseño, se debe comprender la relación sensible que existe entre el usuario y el lugar. Aquí, el concepto de relación sensible con el espacio se utiliza para entender la relación sensorial de los habitantes con su entorno (Thibaud 2001). La UMAG elaboró a través de su Dirección de Infraestructura un Plan Maestro para su campus en la ciudad de Punta Arenas, y solicitó la elaboración de un estudio y diagnóstico que recogiera la información necesaria para la propuesta de diseño urbano. Desde un punto de vista metodológico, el estudio diagnóstico que precede el diseño del Plan Maestro se entiende como un proceso técnico y un proceso participativo. El proceso participativo tenía como fin incorporar aspectos sobre la identidad, la experiencia y la percepción, detectar los principales problemas y necesidades de la comunidad universitaria, así como involucrarla en la toma de decisiones funcionales del instrumento y mantenerla informada de los avances del estudio.

Con ese objetivo, se decidió realizar una metodología de participación a partir de la aplicación de tres herramientas complementarias: 1) diez reuniones o grupos focales sostenidos con la diversidad de actores (informantes clave) de la comunidad universitaria, intentando representar los diferentes intereses; 2) despliegue por un día completo de un plano de la universidad, donde de manera libre y anónima los diversos usuarios eran invitados a responder cuatro preguntas y localizar sus respuestas dentro del plano del campus con un post-it; y 3) recorrido comentado con un grupo de usuarios del campus. Es importante subrayar que la metodología de recorridos comentados, por su carácter situado, exige para su aplicación, ser revisada y adaptada a cada problema y territorio particular en el cual se aplique. Se debió reflexionar en torno a la definición de los recorridos, a la configuración de los grupos de participantes, y a los tiempos y momentos del recorrido. Este artículo busca ahondar en torno a los resultados obtenidos a partir de la tercera herramienta utilizada. 


\section{EL CAMINAR COMO METODOLOGÍA PARA APRENDER LO URBANO.}

\section{Nosotros nos interesamos en la caminata}

como herramienta de exploración con fines cognitivos y críticos sobre el espacio, así como un dispositivo de participación ciudadana. El particular enfoque cognitivo del relato en la medida que se recorre un lugar nace de acercamientos anteriormente emprendidos por antropólogos, escritores, poetas y cineastas. Los situacionistas fueron los primeros que utilizaron las llamadas 'derivas' como una forma de recopilar aspectos de la ciudad para la construcción de sus mapas psico-geográficos o sus unidades de ambiente (Debord 1975). Sin embargo, los estudios en base a la práctica caminatoria son diversos. El elogio poético al caminante realizado por Sansot (2004) - la famosa figura del flanêur de Baudelaire que Walter Benjamin (2005) teoriza en el contexto de la ciudad de París, son algunas muestras de la pertinencia del andar como método de observación de lo urbano. En el mundo anglosajón, con el objetivo de poder concluir cómo los habitantes de la ciudad contemporánea percibían las formas urbanas y sus transformaciones, el antropólogo Kevin Lynch hacía caminar a los habitantes para activar sus percepciones y sentidos (Lynch 1959). La socióloga alemana Margareth Kusenbach, mientras tanto, ha desarrollado y teorizado la metodología conocida como go-along como una herramienta etnográfica híbrida entre observación participante y entrevista cualitativa (Kusenbach 2003) Este método busca explorar el rol de los lugares en la experiencia cotidiana, a través del acompañamiento de los habitantes por medio de un informante en sus itinerarios cotidianos, al mismo tiempo que se entrevista y observa. Kusenbach, por ejemplo, estudió cómo los habitantes de dos barrios urbanos de Hollywood percibían sus problemas locales y cómo dicha percepción se relacionaba con sus actividades e interacciones sociales.

En el contexto político e intelectual de mediados del siglo XX en Francia y Alemania, se buscaba una legitimación de una sociología de terreno atenta a la vida cotidiana, a la praxis (Lefebvre 1999), y alejada de las teorías globales?, poniendo en evidencia aspectos físicos de la realidad, pero también sus aspectos fenomenológicos, imaginarios y sensibles (Grafmeyer 1979). Uno de los primeros sociólogos que incorporó la práctica caminatoria en la investigación de los espacios urbanos fue Jean-François Augoyard, quien construyó una retórica caminatoria a través del estudio en un barrio en Grenoble, Francia. Esta retórica caminatoria sería capaz de traducir "la organización de los estilos propios a cada habitante y las correlaciones entre estos estilos al interior de un espacio común" (Augoyard 1979: 28). Inspirándose en su obra, se crea en 1979 el laboratorio de investigación CRESSON (Centre de recherche sur l'espace sonore \& l'environnement urbain) en Grenoble, Francia. Los trabajos de CRESSON sobre l'itineraire (Petiteau y Pasquier 2001) o le parcours commenté (Thibaud 2001), dan cuenta de la proyección de esta línea de investigación. El itinerario es un método de investigación in situ, desarrollado en primera instancia por Jean-Yves Petiteau, que a diferencia del recorrido comentado o parcours commenté, consiste en una entrevista en terreno donde el entrevistado propone el recorrido y guía al investigador por el lugar de estudio. Petiteau y Pasquier describen el método del itinerario como una transacción, una experiencia compartida entre investigador y entrevistado. De esa forma, el itinerario se configura como un desplazamiento por el territorio y por su universo de referencias.

El andar como herramienta de conocimiento crítico nace como un modo de observar el paisaje desde el movimiento. Las transformaciones de la ciudad contemporánea implican una sensibilidad mayor con respecto a las investigaciones urbanas. En palabras de Careri: "el hecho de atravesar, instrumento de conocimiento fenomenológico y de interpretación simbólica del territorio, es una forma de lectura psicogeográfica del territorio..." (1996: 427). Francesco Careri, postula el andar como práctica estética que dibuja el trazado de los asentamientos humanos, traduciéndose como la primera acción humana que implica un ordenamiento a su alrededor. La constante mutación de las ciudades es producto del movimiento de personas y sus variaciones, y esto nos traslada a una posible comprensión de la ciudad desde una contraposición de la mirada estática (Careri 2002). En palabras del mismo autor: "a través del andar el hombre empezó a construir el paisaje natural que lo rodeaba. Y a través del andar se han conformado en nuestro siglo las categorías con las cuales interpretamos los paisajes urbanos que nos rodean" (Careri 2002: 19-20). El andar revela así el espacio desde el dinamismo individual y social de una ciudad. De aquí se desprende la necesidad imprescindible del movimiento para entender las relaciones que ha forjado el hombre con el territorio.

Estas herramientas utilizadas en la investigación han ido permeando la práctica de los urbanistas en Francia a través de su incorporación en los diagnósticos urbanos. Este tipo de trabajos profesionales han sido desarrollados por el Centre Scientifique et Technique du Bâtiment, mediante la aplicación del diagnostic en marchant; por el colectivo Bazar Urbain, con la aplicación de parcours commentés; y por el Atelier d'Urbanisme de Paris, con las marches exp/oratoires para el estudio de espacios urbanos y arquitectónicos. Estos casos desarrollan experiencias que se inscriben, por una parte, en el diagnóstico o estudio de los espacios y, por otra, en una dinámica de participación ciudadana y/o de coproducción del espacio. Nuestro estudio se inscribe en esta línea de trabajo, adaptando la herramienta de recorridos comentados al caso particular de un diagnóstico de un campus universitario. 


\section{UNA EXPERIENCIA CAMINATORIA POR UN CAMPUS UNIVERSITARIO: Una metodología de investigación} en movimiento. El campus de la UMAG

se ubica al norte de la ciudad de Punta

Arenas, sector que se caracteriza por

ser un área de transición entre el área periurbana norte y el centro. Esta zona limita con grandes predios productivos y de equipamiento que conviven con áreas residenciales en consolidación. La mayor parte de las dependencias de la UMAG se encuentra distribuida entre el Campus Norte y el Instituto de la Patagonia (I.D.P). Sin embargo, el grado de consolidación y de ocupación del campus es bajo. El campus donde se desarrolla este estudio abarca dos predios, ubicados uno frente al otro, y separados por la Avenida Presidente Manuel Bulnes, una de las principales arterias de conexión norte-sur de la ciudad. La falta de una imagen urbana común, sumada al amplio perfil de la avenida, dificulta la conexión y relación simbólica entre ambos campus (figura 1).

El recorrido trazado incluyó una primera etapa por el corazón de la vida universitaria, lugar donde los participantes fueron convocados, y luego un itinerario en línea recta de extremo a extremo del campus, comenzando este por el fondo del predio del Campus Norte y terminando en la zona de cultivos del I.D.P. Específicamente, el recorrido cruzaba longitudinalmente el campus, atravesando espacios y situaciones diferentes. Esta decisión de recorrido fue tomada por las investigadoras a partir de la reflexión sobre los resultados que arrojó el proceso previo de participación ciudadana en relación a la escasa e ineficiente conexión entre ambos predios del campus, percibiéndose actualmente como unidades separadas. Esta experiencia fue registrada en su totalidad por la Direccion de Comunicaciones de la UMAG con cámaras de video, cámaras fotográficas y micrófonos, con el fin de tener un registro de las opiniones de cada uno de los participantes y de los lugares visitados (figura 2).

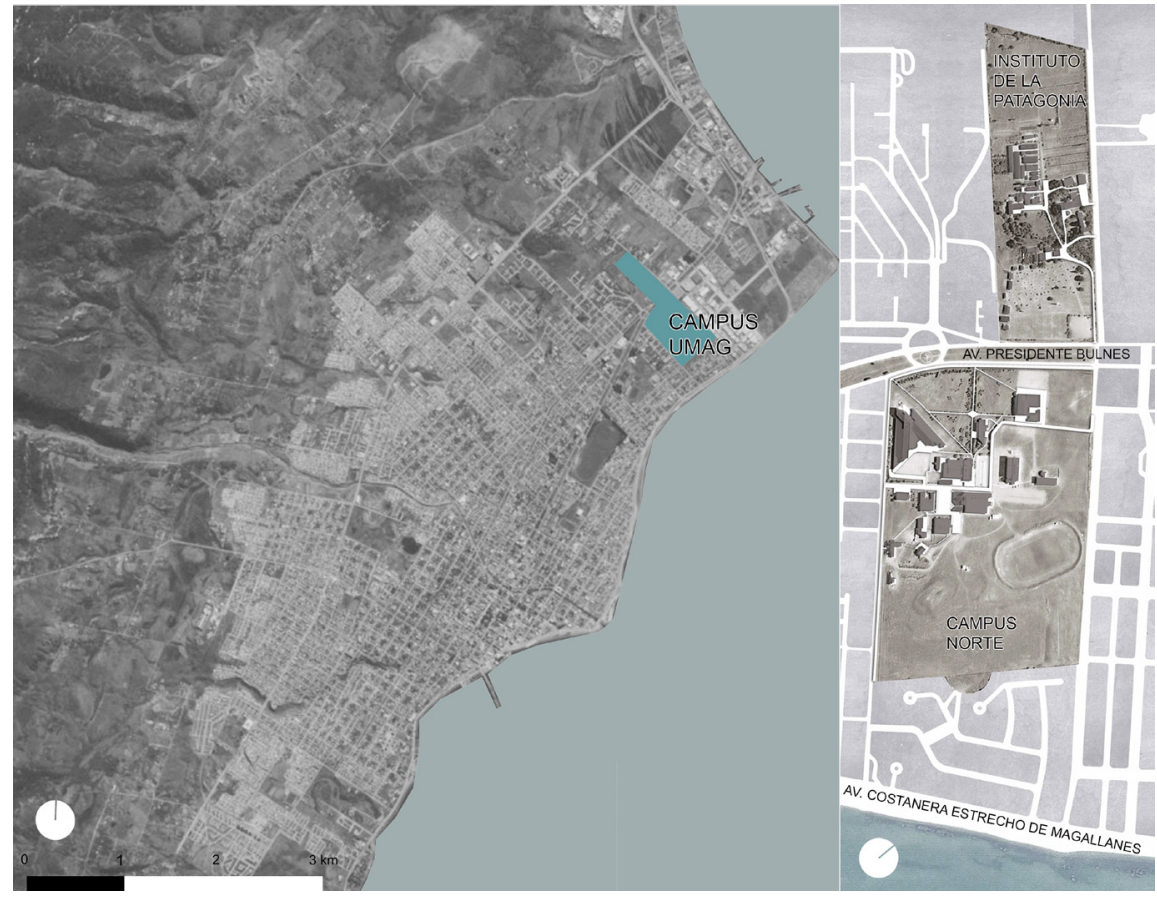

Figura 1. Plano de la ciudad de Punta Arenas. Campus Universidad de Magallanes (fuente: Elaboración propia).

- $1^{\circ}$ etapa: Nos insertamos en una caminata por el corazón de la vida universitaria del campus con el fin de escuchar sus comentarios e impresiones de lo que ven, sienten, escuchan o recuerdan de la experiencia de esos espacios.

$\mathbf{- = - \mathbf { - }} 2^{\circ}$ etapa: Recorreremos una línea no construida, fuera de senderos establecidos, una línea que une la experiencia de los dos campus y en su recorrido comentamos que necesitaría este caminar en términos de comodidad acústica, visual, térmica y de confort para transformarse en un espacio de conexión entre ambos campus.

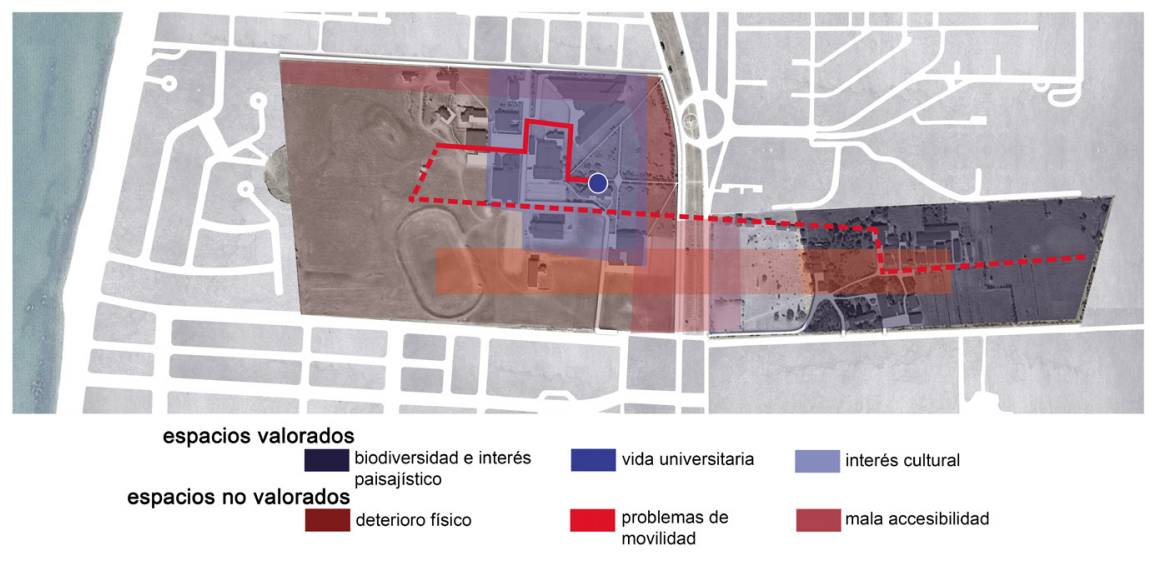

Figura 2. Plano del trazado del recorrido (fuente: Elaboración propia). 

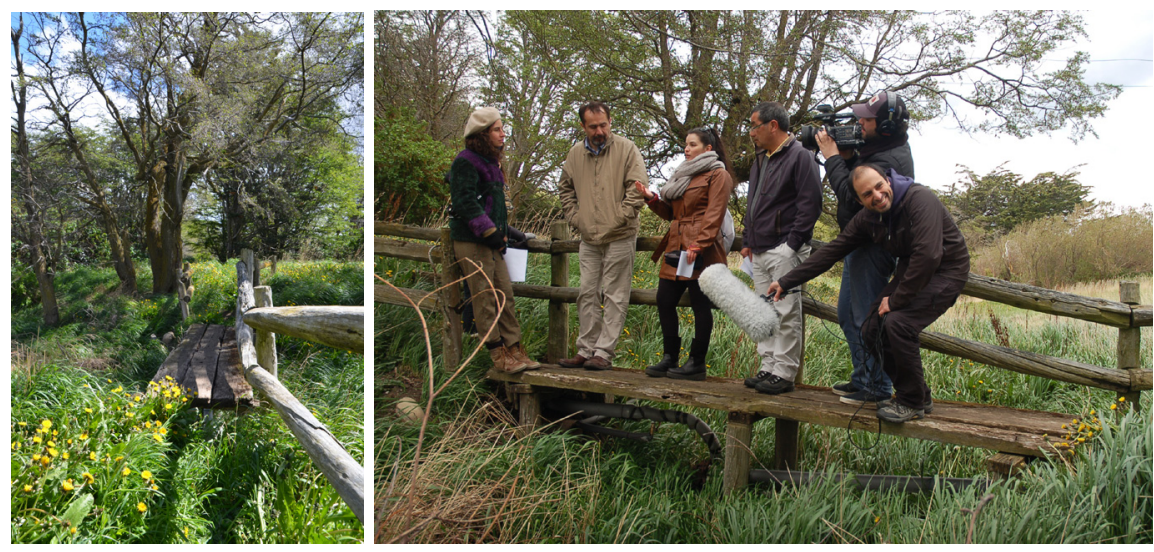

Imagen 1. Fotografía del recorrido por senderos informales (fuente: Las autoras).

La experiencia fue realizada con un solo grupo, conformado por una diversidad de usuarios del campus. Las investigadoras cumplían el rol de monitoras del recorrido, pero también formaban parte del equipo, interfiriendo en momentos particulares donde se detenían en el recorrido para dar la palabra, realizar preguntas y manifestar su propia experiencia como usuarios externos del campus. Incluso los camarógrafos, quienes además eran funcionarios de la universidad y exalumnos de la misma, participaron activamente del recorrido. Esta investigación muestra cómo la experiencia ayuda a generar una sensación de equipo de caminantes que experimentan el lugar en conjunto.

El trazado en línea recta coloca el recorrido como un eje imaginario que unifica ambos campus. La caminata por este eje permite reflexionar en torno a esta segregación y los beneficios de trabajar el diseño desde la unidad, despertando la consciencia de un campus integrado, que permita la movilidad de los estudiantes y académicos a lo largo de toda la universidad. De alguna manera, la experiencia consiste en vivenciar el campus del futuro in situ. Al trazar este eje imaginario, el recorrido incorporó lugares poco frecuentados por los habitantes e, incluso, lugares abandonados. Fue una sorpresa cómo el paso por estos lugares no frecuentados hizo surgir relatos - y despertar la memoria en torno a los mismos. El recorrido comentado permite intencionar la recolección de información del usuario sobre lugares olvidados, pero que despiertan recuerdos y apreciaciones en el contacto sensorial con ellos. Además, el encuentro directo con la realidad, olvidada en las opiniones vertidas durante las reuniones participativas precedentes, - la visita a lugares poco frecuentados, también es de interés, ya que muestra lugares o aspectos del espacio que no son reconocidos o retenidos en la memoria de los usuarios (imagen 1).

Se descubren nuevos paisajes y el usuario adquiere una posición de espectador de su propio espacio que, si bien es utilizado cotidianamente, en este recorrido es observado desde otros ángulos y redescubierto gracias a la predisposición de estar con todos los sentidos atentos al entorno. Algunos paisajes se presentan como escenarios desconocidos y llaman la atención por su belleza. El recorrido desde el museo hacia el jardín botánico es un recorrido por senderos no formalizados, lugar donde los participantes reconocen la belleza del lugar y la falta de acceso a este sector. Este trayecto genera una sensación colectiva de que la universidad no está ocupando los lugares más bellos que tiene y que habría que adaptar su uso para que estos se integren a la vida universitaria respetando el entorno. Se descubre la naturaleza y se valora ese contacto directo que se tiene con ella dentro del campus. Un ejemplo de esto es el caso de la zona deportiva, donde anidan queltehues, que, con graznidos, defienden sus nidos que se encuentran en los pastizales sobre los cuales nos encontramos caminando (figura 3). Dentro del recorrido se pudo valorizar in situ una serie de capas de información histórica y natural contenida en aquellos lugares poco frecuentados del campus, pero con gran carga simbólica para la universidad. Este es el caso del Museo del Recuerdo, museo histórico de la ciudad de Punta Arenas, que tiene la particularidad de ser un gran museo al aire libre, cuya muestra considera objetos, edificaciones, trenes, carruajes y maquinarias que muestran el proceso de desarrollo de la región y la forma de vida desde la colonización pionera en 1880 hasta 1950. Por otra parte, cabe mencionar un jardín botánico que relata la historia ambiental del lugar, dando lugar a una biodiversidad que aloja especies nativas de flora y fauna (figura 4).

La experiencia del trayecto por una zona de experimentación científica ubicada en el fondo de uno de los predios, permitió también reconocer una parte del trabajo científico de la universidad, desconocido para algunos de quienes hacien el recorrido. Algunos participantes con más años y conocimiento de la historia de la Universidad, nos relatan el valor patrimonial de algunas edificaciones tales como los 

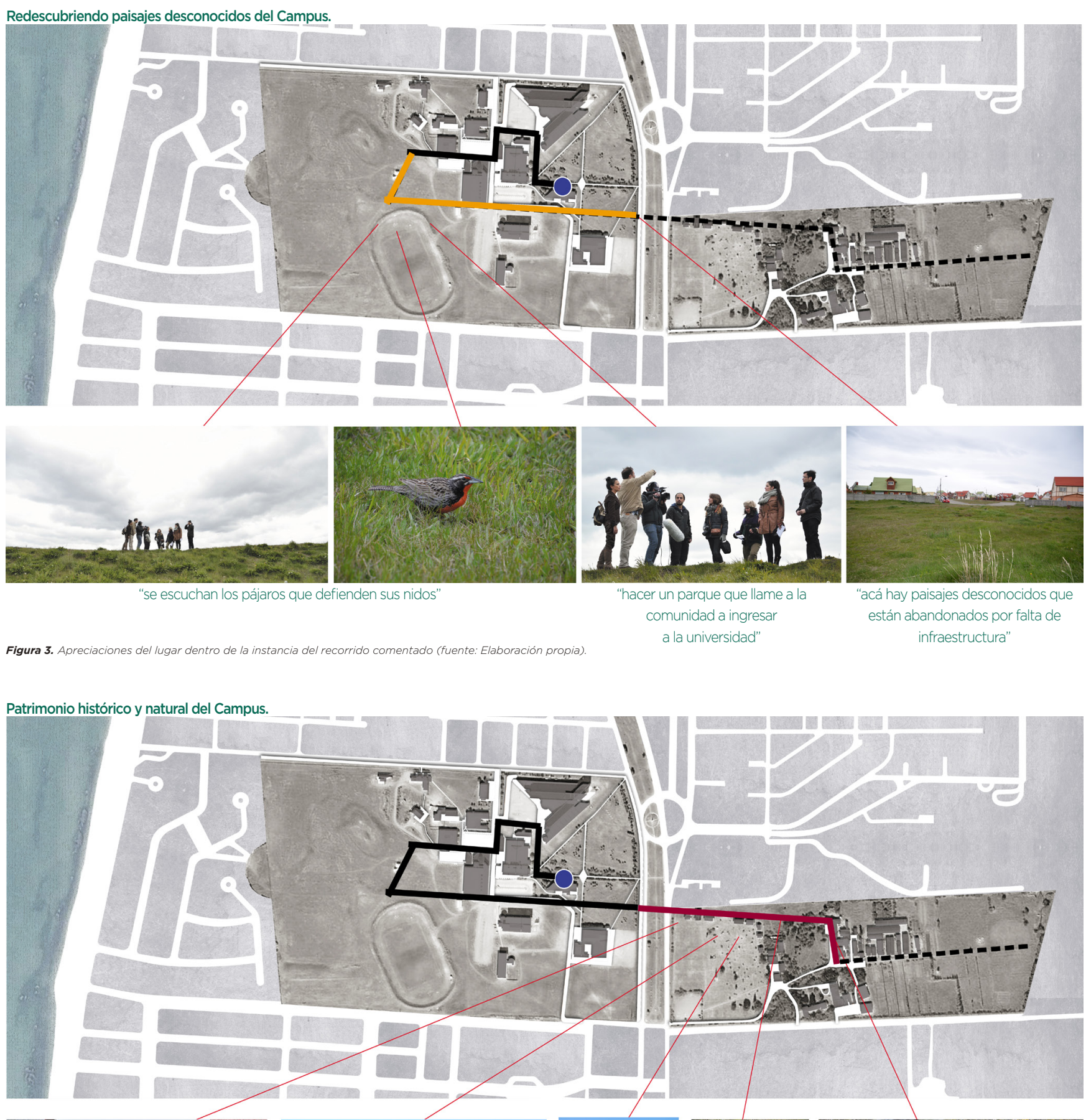

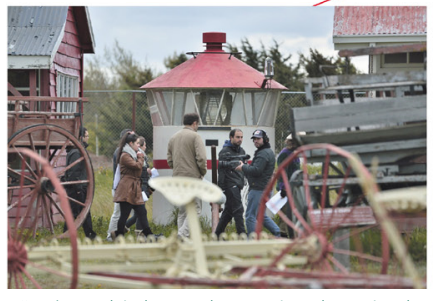

"acá cambia la escala y mejora la actitud hacia el espacio"

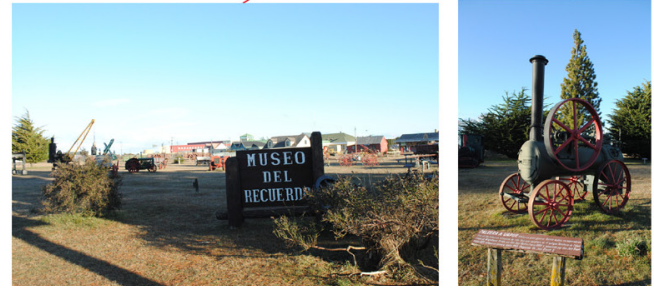

el museo del recuerdo es un espacio que

no es visitado por los estudiantes de manera cotidiana"

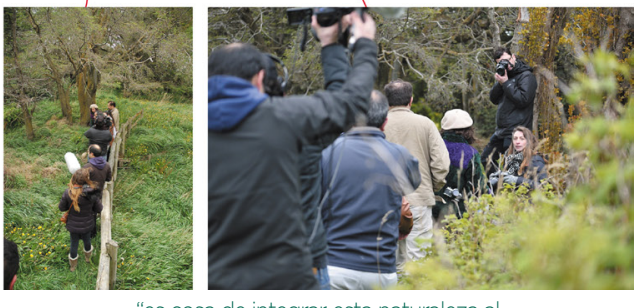

"es cosa de integrar esta naturaleza al campus. Hay que sacar la barrera que existe" 
Zona de exprimentación científica.

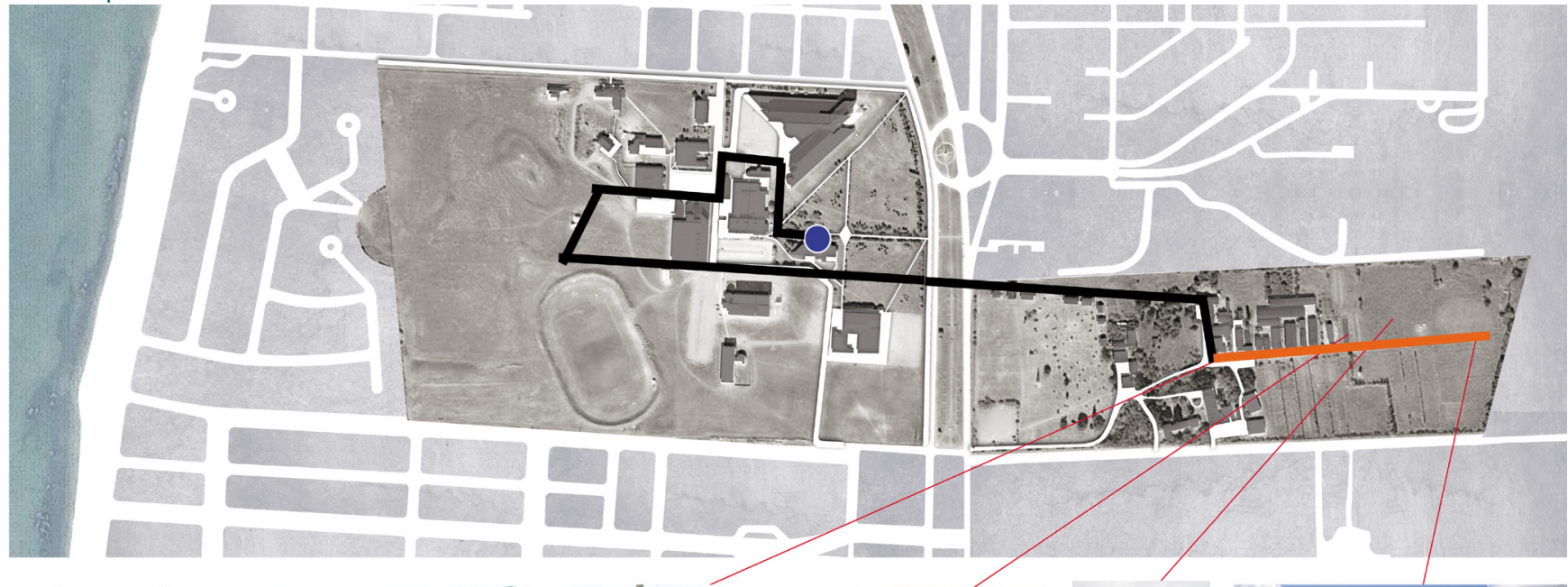

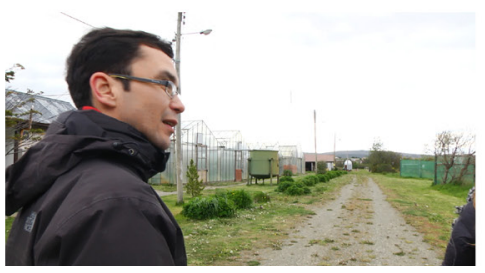

"los invernaderos son elementos icónicos, puesto que son parte de la fundación del Instituto de la Patagonia"

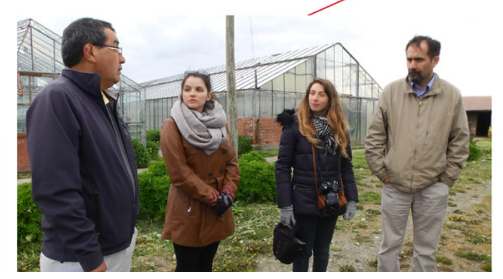

"en otros tiempos os invernaderos tuvieron una gran producción a raiz de la horticultura"

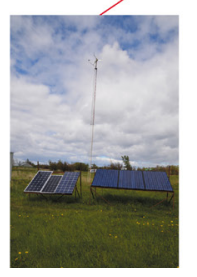

el observatorio tiene un valor patrimonial, sin embargo ahora esta abandonado"

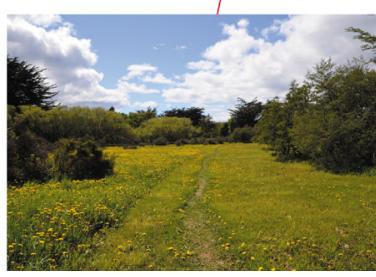

"habría que resaltar la belleza de esta parte del campus"

Figura 5. Apreciaciones del lugar dentro de la instancia del recorrido comentado (fuente: Elaboración propia).

Problemas de diseño dentro del Campus.

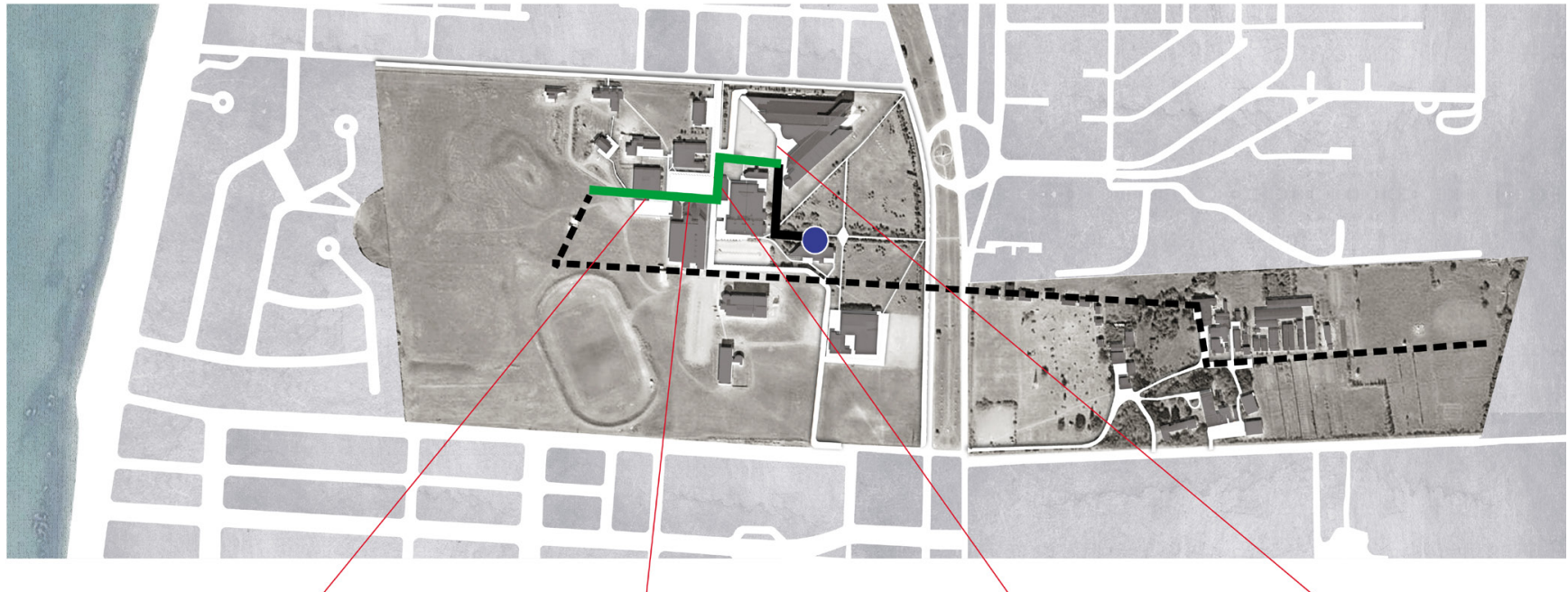

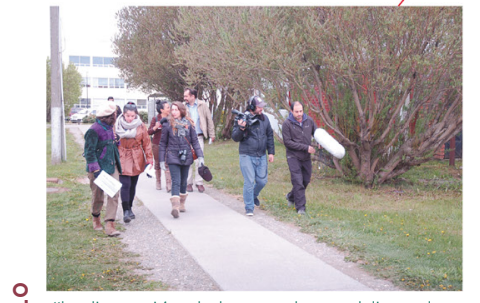

"la dimensión de los senderos obliga a los

ㄴ. $\quad$ alumnos a caminar en fila"

$\stackrel{\alpha}{\alpha}$

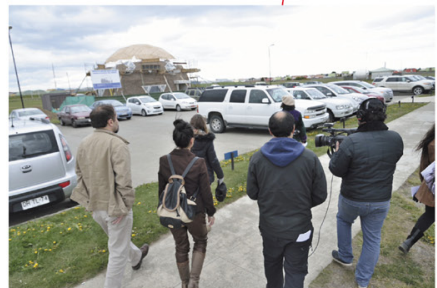

"los autos ocupan los espacios

más importantes"

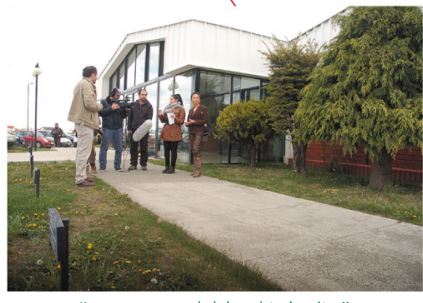

no es agradable al tránsito"

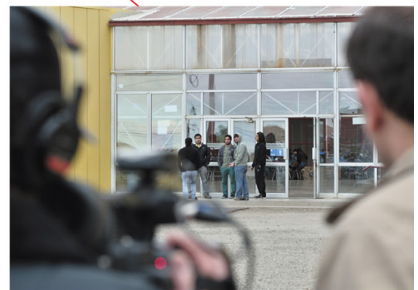

"los alumnos se reunen en el acceso, sin estar diseñado para ello" 
invernaderos presentes en esta área y el observatorio, un elemento que, si bien en este momento está abandonado, perdura en el recuerdo de las personas por lo que fue en el pasado. Además, se destaca una estación meteorológica que funciona hasta el día de hoy (figura 5).

El recorrido no solo evidencia aquellos aspectos desconocidos a valorar, sino que también va delatando una serie de problemas de diseño: pavimentos en mal estado, perfiles mal dimensionados, suelos inundados, etc. Se discutió sobre diversos senderos informales que se han ido formando por el uso de los transeúntes, al ser utilizados como atajos. Se observa el comportamiento de la vida universitaria: grupos de estudiantes, académicos y funcionarios reunidos en diferentes lugares, algunos de los cuales han sido diseñados para el encuentro, mientras otros han sido adaptados o apropiados espontáneamente, como los accesos a algunos edificios (figura 6).

Se abre también la discusión sobre los lugares con condiciones para albergar el acto de reunirse. Se observa un espacio recientemente equipado con la instalación de bancas, las que, sin embargo, no se utilizan regularmente. Se hace el ejercicio de observar y discutir las razones que generan esta obsolescencia. Este tipo de diálogos dentro del recorrido comentado crean una situación en la cual los participantes describen y analizan cómo la comunidad universitaria utiliza y se apropia de los espacios. Esta posición los hace reafirmar y validar apreciaciones hechas anteriormente, pero también los lleva a idear en terreno proyectos para el lugar, tomando el rol de diseñadores.

A partir de esta experiencia, se llevó a cabo un proceso sistemático de traducción de los resultados obtenidos en base a la información sensible del lugar, en formato de apreciaciones personales y colectivas, tendiente a la elaboración de estrategias de diseño para el Master Plan UMAG (tabla 1).
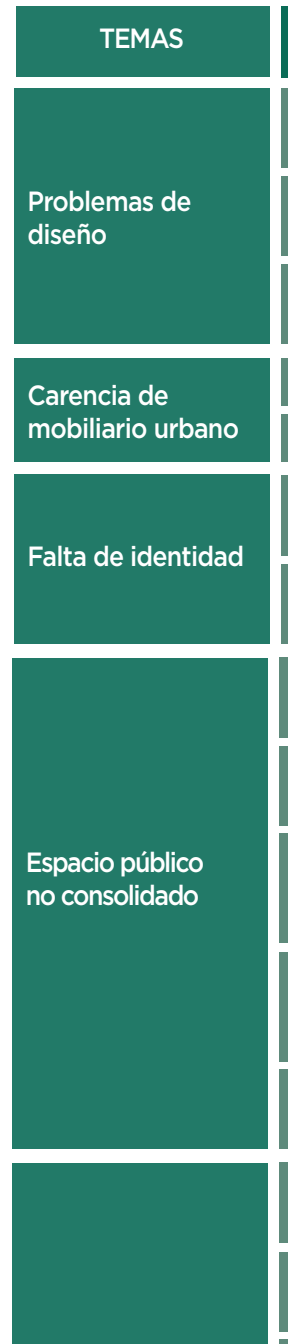

Patrimonio natural
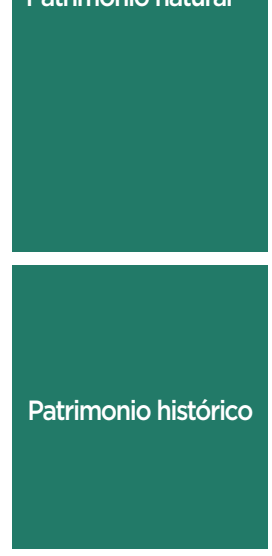

Tabla 1. Análisis de resultados. Tabla de traducción de apreciaciones a estrategias (fuente: Elaboración propia).

\section{ESTRATEGIAS}

Los senderos y aceras son estrechos para
caminar en grupo.
El caminar no es agradable. No hay
continuidad ni suficiente espacio protegido.
Hacia la cancha se pierde cualquier
referencia de camino o sendero.

\begin{tabular}{l}
\hline Espacios públicos sin mobiliarios. \\
\hline Faltan basureros y bancas.
\end{tabular}
Acceso principal tiene poco identificación con
la Universidad.

Faltan referentes simbólicos dentro
del campus.

Se reconocen espacios importantes con potencial de espacio público sin infraestructura.

La plaza de acceso al Edificio de Ingeniería genera una sensación de aridez.

Los espacios no invitan a permanencer y vivir cubiertos para los días de lluvia.

Los autos tienen demasiado protagonismo en los espacios. Habría que ubicar los estacionamientos en otro lugar.

Cada facultad y edificio dentro del campus debiese tener un patio.

Se descubren nidos de queltehues en la zona
de cancha.

Se imaginan un gran parque arborizado en el sector de la cancha.

Una vez que caminamos por el Arboretum frente a Bulnes, se menciona cómo estos espacios verdes logran producir espacios de permanencia.

En el jardín botánico y zona de

experimentación cientifica se descubre un paisaje que no todos conocían y que llama la atención por su belleza.

\section{El recorrido desde el museo hacia el jardin botánico es un recorrido por senderos no formalizados.} Los invernaderos son elementos icónicos de la
historia magallánica.

Se destaca el observatorio y la estación meterológica. al exterior. No existen patios al aire libre ni
Formalización de recorridos. Potenciar la conectividad y

la orientación al interior del campus, entre edificios y zonas universitarias con paseos peatonales de dimensiones coherentes con el flujo de personas que acoge.

Nuevo mobiliario urbano.

Equipar con mobiliario urbano todo el Campus.

Potenciar los elementos identitarios. Recobrar la unidad pasisajistica. Potenciar la imagen del Museo del Recuerdo y consolidar el área de experimentación científica.

Consolidación de espacio público. Reconocer espacios importantes y con potencial de uso público para consolidarlos como espacios públicos que aporten la vida universitaria.

Activación del paisaje. Hacer un parque en cada sector de la Universidad, que integre el paisaje del lugar y sea una plataforma de acceso a aquellos espacios naturales del Campus que por su dificil acceso no son habitados. Este parque debe ser representativo de la flora y fauna local, potenciando los ecosistemas existentes y creando nuevos polos de diversidad.

Activación del patrimonio histórico. Integrar el Museo del Recuerdo y la zona de experimentación científica a la universidad mediante la vinculación directa con estos espacios. 
CONCLUSIONES. El recorrido comentado integra la observación en movimiento como una premisa que conlleva a la reflexión de un escenario cotidiano y sus diferentes lecturas. El método permite al participante tomar diferentes roles ante la acción de los diversos actores que habitan la zona. Es un ejercicio que se traduce en información que solo es posible obtener ante la combinación del habitante con el lugar de estudio, pues esa interacción es la que arroja resultados inéditos para los mismos participantes. El acto de andar permite al entrevistado reflexionar no sobre el espacio en sí, sino sobre la acción misma del andar y acerca de cómo este acto configura el espacio circundante. Se vela por la importancia del recorrido mismo, que abre la discusión sobre las cualidades de este como un espacio imprescindible para la conexión entre los espacios de permanencia, los tiempos, distancias, dimensiones y características del trayecto. La metodología detona una mirada crítica sobre la movilidad y el sistema de ordenamiento que implica el desplazamiento.

Por otro lado, el método estimula la imaginación. No solo se trata de una caminata por el lugar de estudio, sino que también es una instancia creativa. En la experiencia del diseño del Plan Maestro UMAG, la propuesta del eje invitaba a los participantes a imaginar un nuevo corredor que uniría ambos campus. La acción de caminar por este corredor gatilló una acción propositiva por parte de los entrevistados, imaginando un bulevar que unificaba las diferentes zonas universitarias. Existió un intercambio de roles entre entrevistado y diseñador, que permitió recoger vivencias y propuestas de la nueva universidad. La palabra es detonada por la experiencia de la percepción in situ, la cual gatilla una reflexión de lo existente, que se traduce en antecedentes para imaginar propuestas. La metodología es eficiente, al generar resultados de calidad, ya que la observación en movimiento genera un diálogo coherente entre lugar y proyecto que da vida a una planificación consciente del contexto. $\mathbf{\Lambda}$

\section{REFERENCIAS}

Augoyard, J., 1979. Pas à pas. Essai sur le cheminement quotidien en milieu urbain. Paris: Ediciones du Seuil. Benjamin, W., 2005. Libro de los Pasajes. Madrid: Ediciones Akal.

Careri, F., 1996. Rome archipel fractal. Voyage dans les combles de la ville. Paris: Techniques \& Architecture № 427.

Careri, F., 2002. Walkscapes. Barcelona: Editorial Gustavo Gili.

Debord, G., 2006. "Theory of the Dérive." En Knabb, K. (Ed.), Situationist International Anthology, Berkeley. Bopsecrets, 62-66,

Gibson, J., 1979. The Ecological Approach to Visual Perception. Nueva Jersey: Lawrence Erlbaum Associates Grafmeyer, Y. y Joseph, I., 1979. L'École de Chicago. Naissance de l'écologie urbaine. Seyssel: Champ Vallon. Grosjean, M. y Thibaud, J., 2001. L'espace urbain en méthodes. Marsella: Ediciones Parenthèses.

Kusenbach, M., 2003. "Street phenomenology. The go-along as ethnographic research tool." Etnography, 4 (3): $455-485$.
Lefebvre, H., 1999. El materialismo dialéctico. Buenos Aires: El Aleph. Lynch, K. 1959. "A walk around the block." Landscape, 8, (3): 24-34

Park, R., 1999. La ciudad y otros ensayos de ecología urbana. Barcelona: Ediciones del Serbal. Petiteau, J. y Pasquier, E., 2001. "La méthode des itinéraires: Récits et parcours." En Grosjean, M. y Thibaud, J. (Dir.), L'espace urbain en méthodes. Marsella: Ediciones Parenthèses

Sansot, P., 2004. La poétique de la ville. París: Ediciones Payot.

Thibaud, J., 2001. "La méthode des parcours commentés." En Grosjean, M. y Thibaud, J. (Dir.), L'espace urbain en méthodes. Marsella: Ediciones Parenthèses.

Whyte, W., 2001. The Social Life of Small Urban Spaces. Nueva York: Project for Public Spaces. 